\title{
Strategies for Reducing Myocardial Infarct Size Following STEMI
}

\author{
Valeria Paradies, Mervyn Huan Hao Chan, \\ and Derek J. Hausenloy
}

\subsection{Introduction}

In the context of ST-segment elevation myocardial infarction (STEMI), early and successful myocardial reperfusion by primary percutaneous coronary intervention (PPCI) is the most powerful intervention for reducing myocardial infarct (MI) size, preserving left ventricular (LV) systolic function and preventing the onset of heart failure. However, despite continual improvements in acute care, the mortality and morbidity rates following STEMI remain significant, with $7 \%$ death and $22 \%$ rehospitalisation for heart failure, at 1 year. Although timely reperfusion of the occluded vessel by PPCI is essential to minimise the acute ischaemic time (a major

\footnotetext{
V. Paradies

National Heart Research Institute Singapore, National Heart Centre, Singapore, Singapore

M. H. H. Chan

National Heart Research Institute Singapore, National Heart Centre, Singapore, Singapore

Cardiovascular and Metabolic Disorders Program, Duke-National University of Singapore Medical School, Singapore, Singapore

D. J. Hausenloy $(\bowtie)$

National Heart Research Institute Singapore, National Heart Centre, Singapore, Singapore

Cardiovascular and Metabolic Disorders Program, Duke-National University of Singapore Medical School, Singapore, Singapore

Yong Loo Lin School of Medicine, National University Singapore, Singapore, Singapore

The Hatter Cardiovascular Institute, Institute of Cardiovascular Science, University College London, London, UK
}

The National Institute of Health Research, University College London Hospitals Biomedical Research Centre, London, UK

Barts Heart Centre, St Bartholomew's Hospital, London, UK

e-mail: derek.hausenloy@duke-nus.edu.sg 
determinant of MI size) and salvage viable myocardium, the process of reperfusion itself, paradoxically, induces additional myocardial injury and cardiomyocyte death, a phenomenon which has been termed 'myocardial reperfusion injury'. This has been shown to contribute to up to $50 \%$ of the final MI size, making it an important therapeutic target to reduce MI size in STEMI patients reperfused by PPCI. However, the translation of novel cardioprotective strategies for targeting myocardial reperfusion injury to reduce MI size following STEMI has been both challenging and disappointing. In this chapter, we provide an overview of myocardial reperfusion injury, discuss the challenges facing the investigation of treatment strategies for reducing MI size following STEMI and highlight future cardioprotective therapies for potentially improving clinical outcomes in STEMI patients.

\subsection{Myocardial Reperfusion Injury: A Residual Target for Cardioprotection in STEMI}

There is currently no effective therapy for reducing myocardial reperfusion injury, making it an important therapeutic target for reducing MI size following PPCI in STEMI patients. It is characterised by:

1. Reperfusion arrhythmias - these are often short-lived and easily treated.

2. Myocardial stunning - this is reversible impairment in myocardial contractile function and is due to oxidative stress and calcium dysregulation.

3. Microvascular obstruction (MVO) - this manifests as coronary no-reflow at the time of PPCI and refers to the inability to perfuse the myocardium at the level of the coronary microvasculature, despite a patent epicardial coronary artery. It has a multifactorial aetiology which includes coronary microembolisation, platelet activation and external compression of coronary microvasculature by swollen endothelial cells and cardiomyocytes. In severe cases of MVO, damage to the endothelium allows the extravasation of red blood cells into the myocardium (termed 'intramyocardial haemorrhage' or IMH). Both MVO and IMH have been shown to be independent predictors of adverse LV remodelling and clinical outcomes following STEMI.

4. Cardiomyocyte death-this lethal form of myocardial reperfusion injury contributes to the final MI size and is due to several factors including abrupt changes in intracellular $\mathrm{pH}$, ATP depletion, oxidative stress, mitochondrial and cytosolic calcium overload, cardiomyocyte hypercontracture and mitochondrial dysfunction.

A number of other proponents also contribute to the pathophysiology of myocardial reperfusion injury, and these include endothelial dysfunction, platelet activation and inflammation. The strongest evidence for the existence and importance of myocardial reperfusion injury is the demonstration that acute MI size can be reduced by applying an intervention solely at the onset of reperfusion. However, given the complex pathophysiology underlying myocardial reperfusion injury, and the fact it manifests in the first few minutes of reperfusion, it is probably no surprise that it has 
been challenging to target reperfusion injury to reduce MI size following STEMI (see later section).

\subsection{Strategies for Targeting Reperfusion Injury to Reduce MI Size Following STEMI}

A large number of treatment strategies, shown to be effective in the experimental setting, have been tested in the clinical setting of STEMI over the years, and many have failed to show any benefit in terms of reducing MI size and/or improving clinical outcomes (Table 22.1), and the reasons for this are discussed in a later section. In this section, we review the most recent interventions which have been tested as cardioprotective strategies for reducing MI size in STEMI patients (Table 22.1). These can be divided into mechanical interventions and pharmacological therapies-the signalling pathways these cardioprotective therapies target in the cardiomyocyte are depicted in Fig. 22.1.

Table 22.1 Major recent clinical trials investigating strategies to reduce myocardial infarct size in STEMI patients

\begin{tabular}{|c|c|c|c|c|}
\hline Treatment & Study & \begin{tabular}{|l} 
Patients \\
$(n)$
\end{tabular} & Endpoints & Results \\
\hline $\begin{array}{l}\text { Ischaemic } \\
\text { postconditioning }\end{array}$ & Staat et al. & 30 & $\begin{array}{l}\text { Infarct size (72-h } \\
\text { AUC total CK) }\end{array}$ & $\begin{array}{l}36 \% \text { reduction in } \\
\text { infarct size in IPC } \\
\text { group }\end{array}$ \\
\hline $\begin{array}{l}\text { Ischaemic } \\
\text { postconditioning }\end{array}$ & $\begin{array}{l}\text { Hofsten et al. } \\
\text { (DANAMI-3) }\end{array}$ & 2000 & $\begin{array}{l}\text { Composite of } \\
\text { death from any } \\
\text { cause and } \\
\text { hospitalisation } \\
\text { for HF at } 2 \text { years }\end{array}$ & $\begin{array}{l}\text { No difference in } \\
\text { primary endpoint }\end{array}$ \\
\hline $\begin{array}{l}\text { Remote ischaemic } \\
\text { conditioning }\end{array}$ & Soth et al. & 333 & $\begin{array}{l}\text { Composite of } \\
\text { all-cause } \\
\text { mortality, MI, } \\
\text { readmission for } \\
\text { HF and } \\
\text { ischaemic } \\
\text { stroke/TIA }\end{array}$ & $\begin{array}{l}\text { Significantly lower } \\
\text { primary endpoint in } \\
\text { remote ischaemic } \\
\text { conditioning group }\end{array}$ \\
\hline $\begin{array}{l}\text { Remote ischaemic } \\
\text { conditioning }\end{array}$ & \begin{tabular}{|l|} 
CONDI-2/ \\
ERIC-PPCI \\
NCT01857414 \\
NCT02342522
\end{tabular} & 4300 & $\begin{array}{l}\text { Composite of } \\
\text { cardiac death } \\
\text { and } \\
\text { hospitalisation } \\
\text { for HF at } \\
12 \text { months }\end{array}$ & Ongoing \\
\hline Hypothermia & $\begin{array}{l}\text { Erlinge et al. } \\
\text { CHILL-MI }\end{array}$ & 120 & $\begin{array}{l}\text { Infarct size } \\
\text { (4 days of CMR) }\end{array}$ & $\begin{array}{l}\text { No differences in } \\
\text { infarct size between } \\
\text { the two groups } \\
\text { Subgroup analysis } \\
\text { showed smaller MI in } \\
\text { early presenter }(<4 \mathrm{~h}) \\
\text { with anterior STEMI }\end{array}$ \\
\hline
\end{tabular}


Table 22.1 (continued)

\begin{tabular}{|c|c|c|c|c|}
\hline Treatment & Study & $\begin{array}{l}\text { Patients } \\
(n)\end{array}$ & Endpoints & Results \\
\hline Statins & $\begin{array}{l}\text { Lyu et al. } \\
\text { Meta-analysis }\end{array}$ & 1058 & $\begin{array}{l}\text { Myocardial } \\
\text { perfusion } \\
\text { measured by } \\
\text { angiographic } \\
\text { parameters } \\
\text { (cTFC and } \\
\text { MBG) }\end{array}$ & $\begin{array}{l}\text { Significantly lower } \\
\text { cTFC and trend } \\
\text { towards lower MBG in } \\
\text { statin arm }\end{array}$ \\
\hline Adenosine & Ross et al. & 2118 & $\begin{array}{l}\text { Infarct size } \\
\text { Composite of } \\
\text { CHF, } \\
\text { rehospitalisation } \\
\text { for CHF and } \\
\text { death at } \\
6 \text { months }\end{array}$ & $\begin{array}{l}\text { IS reduction in } \\
\text { adenosine group } \\
\text { No difference in } \\
\text { clinical endpoint }\end{array}$ \\
\hline $\begin{array}{l}\text { Adenosine vs. } \\
\text { nitroprusside vs. } \\
\text { placebo }\end{array}$ & $\begin{array}{l}\text { Nazir et al. } \\
\text { REFLO- } \\
\text { STEMI }\end{array}$ & 247 & $\begin{array}{l}\text { Infarct size and } \\
\text { MVO (1-4 days } \\
\text { of CMR) } \\
\text { Angiographic } \\
\text { measures } \\
\text { Composite of } \\
\text { death, TLR, } \\
\text { recurrent MI, } \\
\text { severe HF and } \\
\text { cerebrovascular } \\
\text { events at } \\
1 \text { month }\end{array}$ & $\begin{array}{l}\text { High-dose } \\
\text { intracoronary } \\
\text { adenosine and } \\
\text { nitroprusside did not } \\
\text { reduce infarct size or } \\
\text { MVO measured by } \\
\text { CMR } \\
\text { Adenosine may } \\
\text { adversely affect } \\
\text { mid-term clinical } \\
\text { outcome }\end{array}$ \\
\hline Cangrelor & $\begin{array}{l}\text { PITRI trial } \\
\text { NCT03102723 }\end{array}$ & 210 & $\begin{array}{l}\text { Infarct size } \\
\text { ( } 3 \text { days of CMR) }\end{array}$ & Ongoing \\
\hline Cyclosporine & Piot et al. & 58 & $\begin{array}{l}\text { Infarct size } \\
\text { (6 months of } \\
\text { CMR) }\end{array}$ & $\begin{array}{l}\text { Reduction in infarct } \\
\text { size in cyclosporine } \\
\text { group }\end{array}$ \\
\hline Cyclosporine & $\begin{array}{l}\text { Latini et al. } \\
\text { CYCLE }\end{array}$ & 410 & $\begin{array}{l}\text { ST-segment } \\
\text { resolution } \geq 70 \% \\
1 \mathrm{~h} \text { after PPCI }\end{array}$ & $\begin{array}{l}\text { No differences in } \\
\text { primary endpoint } \\
\text { between the two } \\
\text { groups }\end{array}$ \\
\hline Cyclosporine & $\begin{array}{l}\text { Cung et al. } \\
\text { CIRCUS }\end{array}$ & 970 & $\begin{array}{l}\text { Composite } \\
\text { endpoint of } \\
\text { death, HF and } \\
\text { adverse LV } \\
\text { remodelling at } \\
1 \text { year }\end{array}$ & $\begin{array}{l}\text { No differences in } \\
\text { clinical outcomes } \\
\text { between the two } \\
\text { groups }\end{array}$ \\
\hline MTP-131 & $\begin{array}{l}\text { Chakrabarti } \\
\text { et al. } \\
\text { EMBRACE- } \\
\text { STEMI }\end{array}$ & 118 & $\begin{array}{l}\text { Infarct size }(72-\mathrm{h} \\
\text { AUC total } \\
\text { CK-MB) }\end{array}$ & $\begin{array}{l}\text { No differences in } \\
\text { primary endpoint } \\
\text { between the two } \\
\text { groups }\end{array}$ \\
\hline TRO40303 & $\begin{array}{l}\text { Atar et al. } \\
\text { MITOCARE }\end{array}$ & 163 & $\begin{array}{l}\text { Infarct size }(72-\mathrm{h} \\
\text { AUC total } \\
\text { CK-MB/ } \\
\text { troponin-T) }\end{array}$ & $\begin{array}{l}\text { No differences in } \\
\text { primary endpoint } \\
\text { between the two } \\
\text { groups }\end{array}$ \\
\hline
\end{tabular}


Table 22.1 (continued)

\begin{tabular}{l|l|l|l|l}
\hline Treatment & Study & $\begin{array}{l}\text { Patients } \\
(n)\end{array}$ & Endpoints & Results \\
\hline Metoprolol & $\begin{array}{l}\text { Roovlink et al. } \\
\text { EARLY BAMI }\end{array}$ & 408 & $\begin{array}{l}\text { Infarct size } \\
(30 \text { days of } \\
\text { CMR })\end{array}$ & $\begin{array}{l}\text { No differences in } \\
\text { primary endpoint } \\
\text { between the two } \\
\text { groups. Metoprolol } \\
\text { group had less } \\
\text { malignant arrhythmias } \\
\text { in the acute phase }\end{array}$ \\
\hline IV sodium nitrite & $\begin{array}{l}\text { Siddiqi et al. } \\
\text { NIAMI }\end{array}$ & 229 & $\begin{array}{l}\text { Infarct size }(72-\mathrm{h} \\
\text { AUC total } \\
\text { CK-MB })\end{array}$ & $\begin{array}{l}\text { No differences in } \\
\text { primary endpoint } \\
\text { between the two } \\
\text { groups }\end{array}$ \\
\hline Inhaled nitric oxide & $\begin{array}{l}\text { NOMI } \\
\text { NCT01398384 }\end{array}$ & 248 & $\begin{array}{l}\text { Infarct size } \\
(2-3 \text { days of } \\
\text { CMR })\end{array}$ & $\begin{array}{l}\text { No differences in } \\
\text { primary endpoint } \\
\text { between the two } \\
\text { groups }\end{array}$ \\
\hline $\begin{array}{l}\text { N-acetylcysteine }+ \text { IV } \\
\text { NTG vs. IV NTG }\end{array}$ & $\begin{array}{l}\text { Pasupathy } \\
\text { et al. } \\
\text { NACIAM }\end{array}$ & 112 & $\begin{array}{l}\text { Infarct size } \\
(3-7 \text { days of } \\
\text { CMR })\end{array}$ & $\begin{array}{l}\text { Infarct size reduced by } \\
30 \% \text { in } \\
\text { N-acetylcysteine + IV } \\
\text { NTG group }\end{array}$ \\
\hline
\end{tabular}
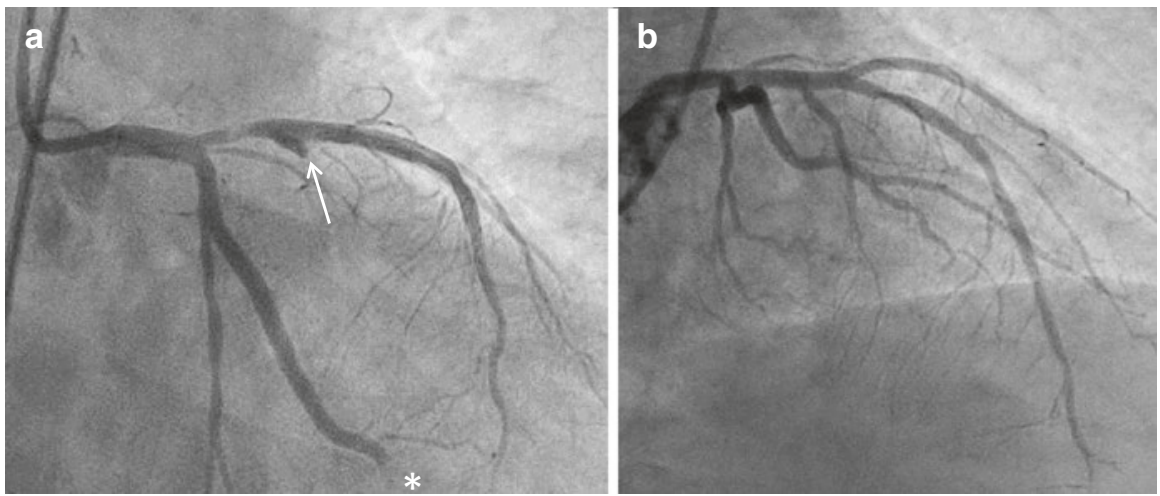

Fig. 22.1 Some of the major signalling pathways underlying ischaemic conditioning and the potential targets of cardioprotective therapies to reduce myocardial infarct size in ST-segment elevation myocardial infarction (STEMI) patients are depicted. The signalling cascade underlying cardioprotection begins at the cardiomyocyte plasma membrane with the activation of G-proteincoupled or cytokine receptors by autocoids such as adenosine, bradykinin or opioids (released in response to the ischaemic postconditioning [IPost] or remote ischaemic conditioning [RIC] stimulus). As a consequence, the reperfusion injury salvage kinase (phosphatidylinositol 3-kinase-Akt (PI3K-Akt) and mitogen-activated protein kinase 1/2-extracellular signal-regulated kinase 1/2 (MEK1/2-Erk1/2)), survivor activator factor enhancement (SAFE), Janus kinase and signal transducer and activator of transcription (JAK-STAT) and the PKG pathways are recruited. These prosurvival signalling pathways activate mediators such as endothelial nitric oxide synthase (eNOS), glycogen synthase kinase (GSK)-3, protein kinase C- $\varepsilon$ (PKC- $\varepsilon$ ) and the mitochondrial ATPdependent potassium channel (KATP), which then mediate an inhibitory effect on mitochondrial permeability transition pore (MPTP) opening 


\subsubsection{Ischaemic Postconditioning: Interrupting Reperfusion to Reduce MI Size Following STEMI}

Experimental studies have demonstrated that following a sustained coronary artery occlusion, interrupting myocardial reperfusion with short-lived episodes of alternating coronary artery occlusion and reflow (four 30-s cycles) in the first few minutes of reflow reduced MI size in small and large animal models of acute myocardial infarction, a phenomenon which has been termed ischaemic postconditioning (IPost). The signalling pathways which mediate the cardioprotective effects of IPost in the cardiomyocyte are depicted in Fig. 22.1.

In the STEMI patient, IPost has been applied at the onset of reperfusion by PPCI using alternating low-pressure inflations and deflations of an angioplasty balloon (four 60-s cycles) positioned upstream of the directly deployed stent within the infarct-related artery, a manoeuvre which has been shown to reduce MI size by $30-40 \%$ and preserve LV systolic function. However, the results of the clinical studies have been mixed with positive, neutral and even negative studies in terms of MI size. Furthermore, two large clinical trials of IPost in STEMI patients were neutral. IPost (four 1-min inflations/deflations of coronary angioplasty balloon following PPCI) failed to improve ST-segment resolution when compared to control in a 700 STEMI patient study, and in the 460 STEMI patient LIPSIA trial, no difference was found between IPost (four 1-min inflations/deflations of coronary angioplasty balloon following PPCI) and control with respect to MRI-determined MI size and clinical outcomes at 6 months (death, re-infarction and new congestive heart failure).

The reasons for the discordant results of IPost in STEMI remain unclear, but they may be due to key factors: patient selection (STEMI patients most likely to benefit are those presenting with a fully occluded coronary artery and treated by direct stenting) and delivery of the IPost protocol (the most effective protocol should be four 60-s angioplasty inflations/deflations, delivered upstream of the deployed stent to avoid potential coronary thromboembolism). The largest clinical study of IPost to date, the multicentre DANAMI 3-iPOST trial, tested the effect of IPost on major clinical endpoints in 1234 STEMI patients treated by PPCI, and it found no difference between IPost (four 30-s inflations/deflations of coronary angioplasty balloon following PPCI) and control with respect to clinical outcomes (all-cause death, re-infarction and hospitalisation for heart failure), after an extended median follow-up of 3.8 years. However, this trial was likely underpowered (the primary event rate was lower than expected), may have used a suboptimal IPost stimulus (four 30-s angioplasty balloon inflations/deflations instead of standard four 60-s protocol) and included patients who were reperfused not using direct stenting.

In summary, IPost in the setting of STEMI is an invasive cardioprotective strategy which requires stringent patient selection and careful delivery of the IPost protocol to be effective. Whether IPost can improve clinical outcomes following STEMI remains unclear. 


\subsubsection{Remote Ischaemic Conditioning: Transient Arm Ischaemia to Reduce MI Size Following STEMI}

IPost requires an invasive intervention to be administered directly to the heart thereby hampering its application in STEMI patients. Therefore, the phenomenon of remote ischaemic conditioning (RIC), which allows the cardioprotective intervention to be applied away from the heart, has greatly facilitated the translation of ischaemic conditioning into the clinical setting. Experimental studies have shown that RIC could be applied to the hindlimb of animals (using a tourniquet to induce cycles of brief ischaemia and reperfusion), and clinical studies have gone on to show that RIC could be non-invasively applied to human volunteers and patients by simply inflating a standard blood pressure cuff placed on the upper arm or thigh to induce cycles of brief ischaemia and reperfusion. The signalling pathways which mediate the cardioprotective effect of RIC in the cardiomyocyte are depicted in Fig. 22.1.

In patients presenting with a STEMI, the application of limb RIC to either the arm or leg prior to or even at the onset of PPCI has been shown in several proof-ofconcept clinical studies to reduce MI size (assessed by serum cardiac biomarkers, myocardial nuclear imaging or cardiac MRI) and preserve LV ejection fraction. Only one recent study has been neutral, but this may have been due to the non-standardised and unorthodox limb RIC protocol used in this study, which comprised variable numbers of cycles of RIC (5 to 115 -min cycles of lower limb cuff inflations/deflations), initiated prior to PPCI and continued to the end of the PCI procedure. Extended follow-up of a cohort of STEMI patients treated by limb RIC reported a 51\% reduction in the combined clinical endpoint of all-cause mortality, non-fatal MI, transient ischaemic attack or stroke and hospitalisation for heart failure, when compared to control, although this study was not originally designed to test the effect of limb RIC on these clinical endpoints. In this regard, the 5200 STEMI CONDI2/ERIC-PPCI patient prospective study is currently investigating whether limb RIC (four 5-min inflations and deflations of a cuff placed on the upper arm administered prior to PPCI) can reduce cardiac death and hospitalisation for heart failure at 12 monthsthe results for this study are expected in the summer of 2019.

In summary, limb RIC provides a non-invasive, low-cost strategy for reducing MI size and has the therapeutic potential to improve clinical outcomes in STEMI patients.

\subsubsection{Therapeutic Hypothermia: Cooling the Heart to Reduce MI Size Following STEMI}

Therapeutic hypothermia has been shown to reduce MI size in preclinical studies by reducing myocardial metabolic demands, preventing platelet aggregation and limiting the inflammatory response. Large animal studies have shown that hypothermia to $32{ }^{\circ} \mathrm{C}$ is cardioprotective if initiated during ischaemia but not at reperfusion. Early clinical studies (ICE-IT, COOL-MI) failed to demonstrate a benefit of 
hypothermia, possibly due to slow cooling. The combination of cold saline and endovascular cooling induced a faster temperature decline and reduced MI size in a 20-patient pilot trial (RAPID MI-ICE). However, the larger CHILL-MI trial failed to demonstrate a significant reduction in MI size, although patients presenting within $4 \mathrm{~h}$ with an anterior STEMI had a reduction in MI size and there was also a significant reduction in heart failure. It was hypothesised, therefore, that faster cooling may be beneficial in anterior STEMI patients. However, the recently published COOL AMI EU Pilot Trial which recruited anterior STEMI patients only $(N=50)$, and used the ZOLL Proteus Intravascular Temperature Management System device to achieve rapid hypothermia $\left(33.6{ }^{\circ} \mathrm{C}\right.$ at the time of PPCI), failed to significantly reduce $\mathrm{MI}$ size, and the intervention resulted in a 17-min delay in reperfusion and increased the rates of paroxysmal atrial fibrillation.

As such, the role of therapeutic hypothermia to reduce MI size following STEMI remains unproven, and it is associated with logistical challenges to implement into the clinical pathway and has side effects.

\subsubsection{Thrombus Aspiration to Improve Microvascular Reperfusion Following STEMI}

Whether routine manual thrombus aspiration at the time of PPCI can reduce distal embolisation and improve microvascular perfusion in STEMI patients has been heavily debated in recent years following positive results in the TAPAS trial (Thrombus Aspiration During Percutaneous Coronary Intervention in Acute Myocardial Infarction Study) which showed that routine thrombus aspiration improved microvascular perfusion and reduced mortality at 1 year. However, two major randomised multicentre trials, TASTE (Thrombus Aspiration in ST-Elevation Myocardial Infarction in Scandinavia) and TOTAL (Trial of Routine Aspiration Thrombectomy with PCI vs. PCI Alone in Patients with STEMI), failed to confirm any beneficial effects of routine thrombus aspiration use during PPCI and found that it may increase the risk of stroke. As a result, the routine use of thrombus aspiration has been changed from a IIa to III recommendation in the latest 2017 ESC STEMI guidelines. Whether selective use of thrombus aspiration in STEMI patients with high thrombus burden is beneficial in terms of improving coronary microvascular perfusion and clinical outcomes remains to be elucidated. Further in-depth discussion of this chapter is available in Chap. 10.

\subsubsection{GLP-1 Agonists to Reduce MI Size Following STEMI}

The anti-diabetic agent, glucagon-like peptide-1 (GLP-1), has been demonstrated in experimental animal studies to reduce MI size when administered at the onset of reperfusion by mechanisms independent of increased insulin levels. As a therapeutic strategy, the GLP-1 analogue, exenatide, has also been shown to protect against myocardial reperfusion injury in small and large animal MI models. In the clinical 
setting, an intravenous infusion of exenatide initiated prior to PPCI has been shown to reduce MI size in patients presenting with an acute STEMI, especially in those patients presenting with short ischaemic times from symptom onset ( $<132 \mathrm{~min}$ ). Another GLP-1 analogue, liraglutide, when administered prior to PPCI and continued for 7 days, has been shown in a study of 85 STEMI patients to improve LV systolic function. However, not all studies have been favourable with neutral effects on MI size in two recently published using exenatide in STEMI patients.

On the basis of these conflicting results, further clinical studies are required to determine whether this therapeutic approach is beneficial as adjunctive therapy to PPCI to reduce MI size in STEMI patients.

\subsubsection{Adenosine to Reduce MI Size Following STEMI}

Experimental studies have demonstrated that adenosine administered prior to index ischaemia can reduce MI size-however, whether it can also reduce MI size when administered at the time of reperfusion has been contentious. Unsurprisingly then, the results of clinical studies investigating adenosine as an adjunct to PPCI have been inconsistent, and this may in part relate to patient selection, the different doses used and the route of administration (intravenous versus intracoronary). Some studies have reported reductions in MI size with high-dose intravenous adenosine administered as a 3-h infusion initiated prior to reperfusion in STEMI patients presenting within $3 \mathrm{~h}$ of chest pain onset, with other studies using lower doses of IV adenosine or boluses of intracoronary adenosine being less successful at reducing MI size. The recently published REFLO-STEMI trial has recently shown that highdose intracoronary adenosine during PPCI did not reduce MI size or MVO measured by cardiac magnetic resonance imaging (CMR) in 247 STEMI patients when compared to conventional treatment. Furthermore, the investigators reported that adenosine may adversely affect mid-term clinical outcomes.

On the basis of these conflicting results, the role of adenosine as adjunctive therapy to PPCI to reduce MI size in STEMI patients remains unclear.

\subsection{Anti-platelet and Anti-thrombotic Strategies to Reduce MI Size Following STEMI}

Anti-platelet and anti-thrombotic agents form the cornerstone of therapy for STEMI patients treated by PPCI. A number of studies have investigated new approaches to optimise anti-platelets and anti-thrombotic therapy to reduce MI size following STEMI.

It has been shown that current oral P2Y12 platelet inhibitors, such as prasugrel and ticagrelor, administered to STEMI patients prior to PPCI, can take 4-6 h to achieve maximal platelet inhibition, meaning that at the time of reperfusion, the platelets are not maximally inhibited-this may be expected to result in an increased risk of MVO and worsened clinical outcomes following STEMI. The 
intravenous (IV) P2Y12 platelet inhibitor, cangrelor, which has a rapid onset of action and induces maximal platelet inhibition within 1-2 min of administration, has been shown in the CHAMPION studies to reduce stent thrombosis and prevent MACE in ACS patients when administered at the time of PCI, when compared to oral clopidogrel. The administration of cangrelor prior to PPCI might be expected to offer complete platelet inhibition at the time of reperfusion, thereby reducing the risk of developing MVO following STEMI. Moreover, a number of experimental small and large animal studies have demonstrated that administrating cangrelor prior to reperfusion has the ability to reduce MI size through a direct cardioprotective effect on the cardiomyocyte involving the activation of established pro-survival signalling cascades. These findings suggest that administering cangrelor prior to reperfusion in STEMI patients has the therapeutic potential to target myocardial reperfusion injury through two distinct mechanisms-maximal platelet inhibition at time of PPCI, thereby preventing MVO, and a direct cardioprotective effect on the cardiomyocyte, thereby reducing MI size. The ongoing multicentre randomised controlled trial "Platelet Inhibition to Target Reperfusion Injury" (PITRI) (ClinicalTrials.gov NCT03102723) is currently investigating whether initiating a cangrelor infusion prior to PPCI can reduce MI size and prevent MVO in STEMI patients treated by PPCI.

The INFUSE-AMI trial demonstrated that an intracoronary bolus of the GPIIb/ IIIa inhibitor, abciximab, reduced MI size on cardiac MRI at 30 days in anterior STEMI patients when compared to placebo, suggesting that potent platelet inhibition delivered locally to the infarct lesion site may be beneficial. Whether administering an intracoronary bolus of abciximab is more effective at reducing MI size and preventing MVO, when compared to using an intravenous bolus, had been unclear. However, the large randomised AIDA-STEMI trial failed to demonstrate significant differences in the primary composite outcome of all-cause mortality, re-infarction and new congestive heart failure between intracoronary and intravenous administration of abciximab. Current European guidelines recommend GPIIb/IIIa inhibitor administration only for bailout, evidence of no-reflow or thrombotic complications.

In order to target the thrombus within the infarct-related coronary artery following PPCI, the ongoing T-TIME trial (Trial of Low-dose Adjunctive alTeplase During prIMary PCI) is currently investigating the effect of low-dose intracoronary alteplase infusion initiated after reperfusion with aspiration thrombectomy \pm angioplasty but before stenting, on the extent MVO on cardiac MRI in STEMI patients treated by PPCI.

\subsection{Targeted Mitochondria to Reduce MI Size Following STEMI}

The immunosuppressant, cyclosporin-A (CsA), has been shown to have a protective effect on mitochondrial function and reduce MI size when administered at the onset of reperfusion in a number of small and large animal experimental studies. Although 
an initial small proof-of-concept studies found that administering an IV bolus of CsA prior to PPCI reduced MI size in STEMI patients treated by PPCI, two subsequent large clinical trials (CYLCE and CIRCUS) failed to find any benefit with this therapeutic approach in terms of ST-segment resolution, enzymatic infarct size and clinical endpoints.

The mitochondria-targeting peptide, MTP-131, which optimises mitochondrial energetics and attenuates the production of ROS by selectively targeting cardiolipin in the inner mitochondrial membrane, has been reported in small and large animal experimental studies to reduce MI size when administered at the onset of reperfusion and prevent adverse LV remodelling following MI. However, in the 117-patient EMBRACE-STEMI clinical trial, intravenous MTP-131 administered prior to PPCI failed to reduce enzymatic MI size in a carefully selected population of anterior STEMI patients with ischaemic time $<4 \mathrm{~h}$, no collaterals and fully occluded coronary artery.

Finally, the mitochondrial targeting drug, TRO40303, which binds to the translocator protein in the outer mitochondrial membrane, has been reported in small animal experimental studies to reduce MI size when administered at time of reperfusion. However, in a clinically relevant large animal MI model, it failed to reduce MI size in the porcine heart. In the 163 STEMI patient MITOCARE study, this agent failed to reduce MI size despite careful patient selection (completely occluded infarctrelated artery, large area-at-risk). Prior experimental studies had revealed ambiguous cardioprotective capacity, and the formulation and dosage of TRO40303 used in the clinical study differed from experimental studies, which may in part explain the neutral findings of the MITOCARE study. Finally, more adverse events were reported in patients receiving TRO40303 when compared to the placebo arm, thereby limiting the clinical application of this therapeutic approach.

As such, the current evidence does not support a role for these three mitoprotective agents as an adjunct to PPCI to reduce MI size following STEMI.

\subsection{Metoprolol: Beta-Blocker Therapy to Reduce MI Size Following STEMI}

Intravenous metoprolol administered prior to reperfusion has been shown to reduce MI size and preserve LV systolic function in the porcine heart. The mechanisms underlying this cardioprotective effect are currently being investigated and appear to extend beyond their effects on haemodynamics and myocardial oxygen consumption. In the 270 anterior STEMI patient METOCARD-CNIC trial, intravenous metoprolol administered in the ambulance prior to PPCI reduced MI size, prevented LV adverse remodelling, preserved LV systolic function and lowered hospital readmissions for heart failure. However, the larger EARLY BAMI trial failed to show any benefit with IV metoprolol on MI size by cardiac MRI or clinical outcomes in a non-selected cohort of 683 STEMI patients.

As such, the current evidence does not support a role for IV metoprolol as an adjunct to PPCI to reduce MI size following STEMI. 


\subsection{Nitric Oxide Donors to Reduce MI Size Following STEMI}

Although there have been experimental studies demonstrating reduced MI size with intravenous nitrite administered at the onset of reperfusion to release nitric oxide, the National Heart, Lung, and Blood Institute (NHLBI) Consortium for preclinicAl assESsment of cARdioprotective therapies (CESAR) Network failed to demonstrate MI size reduction with nitrite using a multicentre approach in small and large animal MI models. Two recent clinical studies have failed to demonstrate a significant reduction in MI size with nitrite administered by either the intravenous or intracoronary routes in STEMI patients treated by PPCI. However, there was a borderline increase in myocardial salvage index and reduced MI size in a subgroup of patients presenting with a fully occluded coronary artery. The 250-patient NOMI study investigated the role of inhaled nitric oxide (vasoKINOX 450) as an adjunct to PPCI to target myocardial reperfusion injury in STEMI patients also failed to reduce MI size (day 3 cardiac MRI), although a post-hoc subgroup analysis revealed that there was a significant reduction in MI size in those patients who had not received nitrates in the ambulance. Recently, the NACIAM trial demonstrated that the coadministration of two old drugs, the nitric oxide donor, nitroglycerin, and the antioxidant, N-acetylcysteine (NAC), reduced MI size on cardiac MRI, but had no beneficial effects on LV remodelling in 112 all-comers STEMI patients.

As such, the current evidence is mixed over whether there is a role for nitric oxide donors as an adjunct to PPCI to reduce MI size following STEMI.

\subsection{Optimising the Translation of Cardioprotection into the Clinical Setting}

Given the numerous examples of failed translation of cardioprotective therapies to reduce MI size and improve clinical outcomes following STEMI, new approaches are required to optimise the preclinical and clinical testing of future cardioprotective therapies - these include the following.

\subsubsection{More Rigorous Preclinical Evaluation of the Cardioprotective Strategy Before Clinical Testing}

A number of clinical trials may have failed to demonstrate benefit with some cardioprotective therapies due to inconsistent and/or insufficient experimental data. In general, most treatment strategies have only been studied in healthy, young animals, and preclinical studies in adult or older animals, with comorbidities and concomitant medication usually received by patients with STEMI, have been lacking. 
Thus, clinical testing of novel cardioprotective strategies should only be performed after consistent demonstration of efficacy and absence of safety concerns obtained in adequate small and large animal models in different laboratories using standardised methods.

\subsubsection{Optimising Clinical Study Design}

(a) Patient selection: It is important to select those STEMI patients who are more likely to benefit from a strategy applied at the onset of reperfusion to reduce MI-this includes those STEMI patients presenting with:

- Short ischaemic time $(<2-3 \mathrm{~h})$

- Large area-at-risk (>30-40\% of LV) such as proximal LAD STEMI

- Fully occluded coronary artery prior to PPCI (TIMI flow <1)

- No significant coronary collaterals

(b) Dosing the intervention: A failure to ascertain the most efficacious dose of the cardioprotective strategy, whether it be a mechanical or pharmacological one, may have contributed to the failure to translate cardioprotection in some of the clinical STEMI studies.

(c) Timing the intervention: There is consistent preclinical evidence that the intervention to target myocardial reperfusion injury might reduce final MI size when administered prior or at the onset of reperfusion, and it has achieved sufficient concentrations in the blood in the first few minutes of reperfusion.

\subsection{Future Perspectives}

Translating cardioprotective therapies for targeting myocardial reperfusion injury to reduce MI size following STEMI has been extremely challenging. The failure to find an effective agent for preventing myocardial reperfusion injury thus far, however, does not question the existence of myocardial reperfusion injury as a valid target for cardioprotection-rather it underscores the need to better understand the mechanisms underlying myocardial reperfusion injury and investigate novel strategies for cardioprotection. These should include combination therapy to (1) target different cardioprotective signalling pathways within the cardiomyocyte in order to provide additive cardioprotection and (2) target the different players involved in myocardial reperfusion injury (cardiomyocyte, microvasculature, inflammatory cells, and platelets). These experimental and clinical studies are currently under way and should allow more effective targeting of myocardial reperfusion injury, thereby reducing MI size in reperfused STEMI and preventing the onset of heart failure. 


\subsection{Case Report: Strategies for Reducing Myocardial Infarct Size Following STEMI}

Valeria Paradies, Mervyn Huan Hao Chan, and Derek J. Hausenloy

\subsubsection{Case Report}

This 41-year-old male with diabetes was referred to our hospital for primary percutaneous coronary intervention (PPCI) for acute onset of chest pain and anterolateral ST-segment elevation myocardial infarction (STEMI) on ECG. He was recruited into the PITRI trial which is currently investigating the effect of an intravenous infusion of the P2Y12 inhibitor, cangrelor, initiated prior to PPCI, on myocardial infarct size and microvascular obstruction (MVO), in STEMI patients treated by PPCI. Right coronary angiography demonstrated the absence of both atherosclerotic lesions and collaterals towards the left coronary artery. Left coronary angiography revealed a critical lesion in the proximal left descending artery (LAD) with evidence of acute thrombus. Distal coronary embolisation and abrupt occlusion of a big septal branch, apical LAD and first marginal branch were detected (Fig. 22.2a). Thrombus aspiration of the septal branch was initially performed with restoration of the flow. The procedure continued with PPCI of the culprit lesion and drug-eluting stent implantation in the proximal LAD. The procedure was completed with thrombectomy of the marginal branch and this achieved good final angiographic result (Fig. 22.2b). Cardiac MRI performed at day 4 after PPCI revealed normal left ventricular (LV) size with severely impaired LV systolic function with akinesia of the LAD territory and mid lateral segment with a dyskinetic apical cap. Late gadolinium enhancement (LGE) images showed transmural infarction in the LAD territory in the basal to mid septum, mid
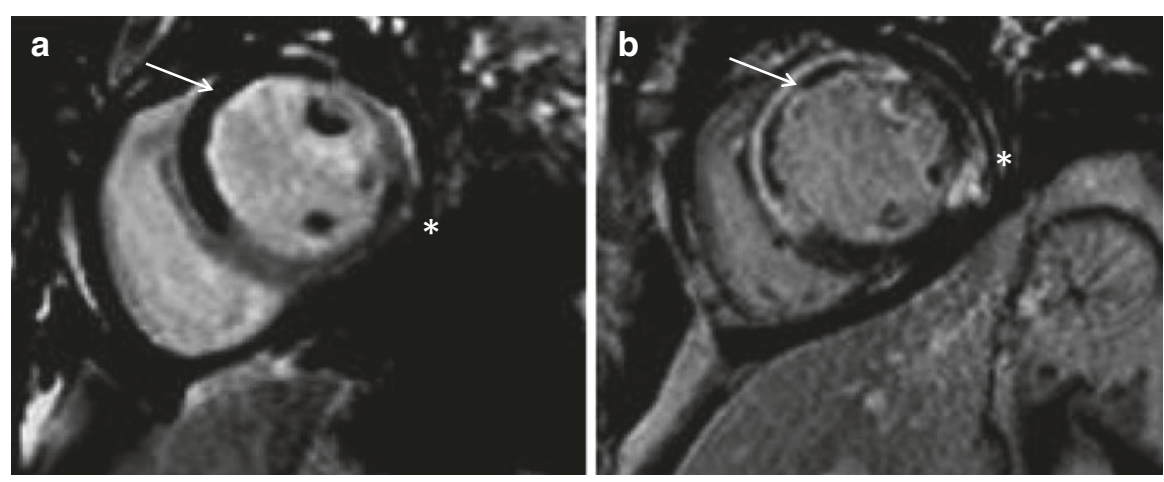

Fig 22.2 Coronary angiogram in acute LAD STEMI patient. (a) Left coronary angiography showing culprit lesion in proximal LAD and distal embolisation in septal branch (arrow), distal LAD and marginal branch (asterisk). (b) Angiographic result after PPCI with proximal LAD, thrombectomy of septal and marginal branches 


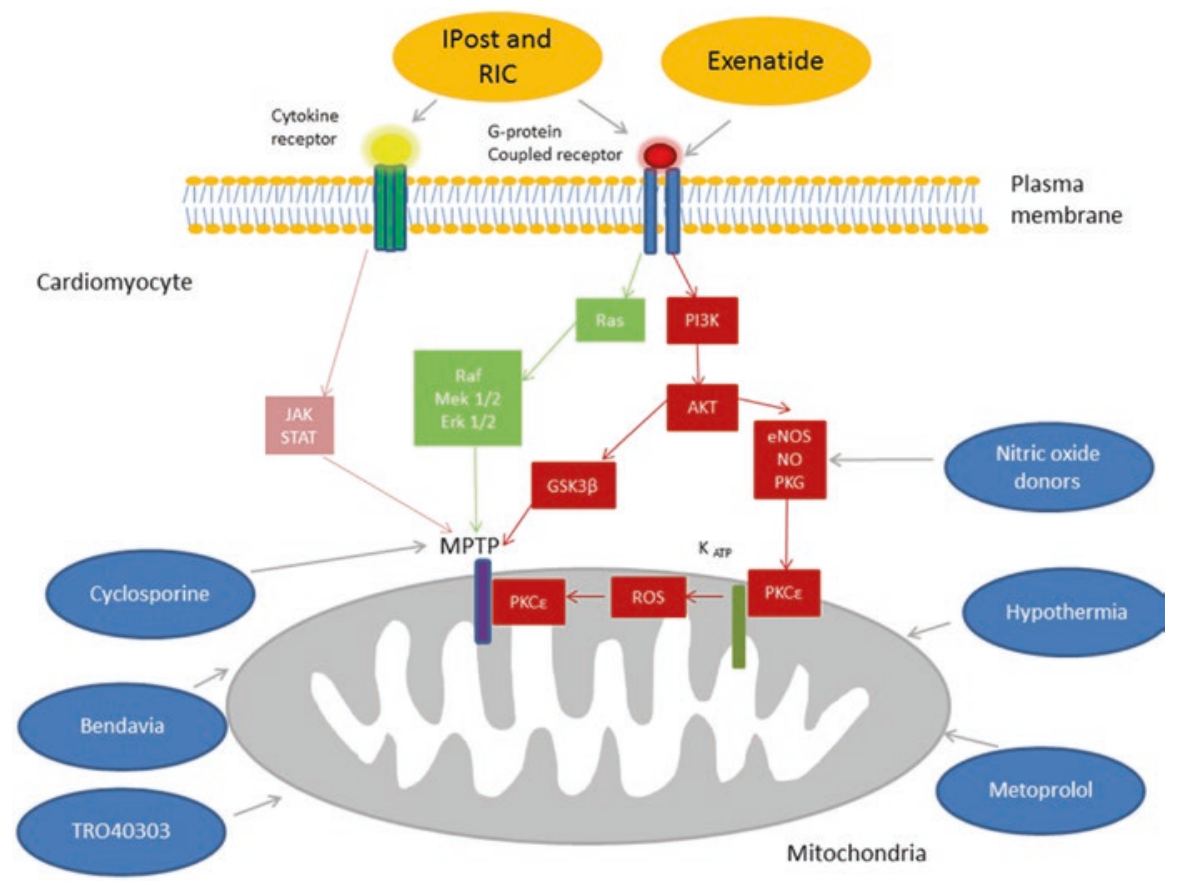

Fig. 22.3 Cardiac MRI scan in acute LAD STEMI patient. (a) Mid LV short-axis slice showing MVO on early gadolinium enhancement (EGE) contrast MRI images in mid septal, anterior (arrow) and lateral LV segments (asterisk). (b) Mid LV short-axis slice showing myocardial infarction on late gadolinium enhancement (LGE) contrast MRI images in mid septal, anterior (arrow) and lateral LV segments (asterisk), with hypointense core representing MVO

anterior, mid lateral and apical segments. MVO was detected at the mid septum and mid lateral LV segments (Fig. 22.3a, b).

This case demonstrates aptly that even with effective and timely reperfusion with PPCI for STEMI, the insult to the myocardium is often substantial, and effective interventions to protect the myocardium from myocardial reperfusion injury (which can contribute up to $50 \%$ of final infarct size) are urgently needed.

\section{Conflicts of Interest None.}

\section{Further Readings}

Fröhlich GM, Meier P, White SK, Yellon DM, Hausenloy DJ. Myocardial reperfusion injury: looking beyond primary PCI. Eur Heart J. 2013;34(23):1714-22.

Hausenloy DJ, Botker HE, Engstrom T, Erlinge D, Heusch G, Ibanez B, Kloner RA, Ovize M, Yellon DM, Garcia-Dorado D. Targeting reperfusion injury in patients with ST-segment elevation myocardial infarction: trials and tribulations. Eur Heart J. 2016;38(13):935-41.

Hausenloy DJ, Garcia-Dorado D, Bøtker HE, Davidson SM, Downey J, Engel FB, Jennings R, Lecour S, Leor J, Madonna R, Ovize M, Perrino C, Prunier F, Schulz R, Sluijter JPG, 
Van Laake LW, Vinten-Johansen J, Yellon DM, Ytrehus K, Heusch G, Ferdinandy P. Novel targets and future strategies for acute cardioprotection: position paper of the European Society of Cardiology Working Group on cellular biology of the heart. Cardiovasc Res. 2017;113(6):564-85.

Hausenloy DJ, Yellon DM. Ischaemic conditioning and reperfusion injury. Nat Rev. Cardiol. 2016;13(4):193-209.

Yellon DM, Hausenloy DJ. Myocardial reperfusion injury. N Engl J Med. 2007;357(11):1121-35.

Open Access This chapter is licensed under the terms of the Creative Commons Attribution 4.0 International License (http://creativecommons.org/licenses/by/4.0/), which permits use, sharing, adaptation, distribution and reproduction in any medium or format, as long as you give appropriate credit to the original author(s) and the source, provide a link to the Creative Commons license and indicate if changes were made.

The images or other third party material in this chapter are included in the chapter's Creative Commons license, unless indicated otherwise in a credit line to the material. If material is not included in the chapter's Creative Commons license and your intended use is not permitted by statutory regulation or exceeds the permitted use, you will need to obtain permission directly from the copyright holder. 